\title{
Scaling Up Cooperative Game Theory Based Energy Management using Prosumer Clustering
}

Han, Liyang; Morstyn, Thomas; McCulloch, Malcolm D.

Published in:

IEEE Transactions on Smart Grid

Link to article, DOI:

10.1109/TSG.2020.3015088

Publication date:

2021

Document Version

Peer reviewed version

Link back to DTU Orbit

Citation (APA):

Han, L., Morstyn, T., \& McCulloch, M. D. (2021). Scaling Up Cooperative Game Theory Based Energy Management using Prosumer Clustering. IEEE Transactions on Smart Grid, 12(1), 289 - 300.

https://doi.org/10.1109/TSG.2020.3015088

\section{General rights}

Copyright and moral rights for the publications made accessible in the public portal are retained by the authors and/or other copyright owners and it is a condition of accessing publications that users recognise and abide by the legal requirements associated with these rights.

- Users may download and print one copy of any publication from the public portal for the purpose of private study or research.

- You may not further distribute the material or use it for any profit-making activity or commercial gain

- You may freely distribute the URL identifying the publication in the public portal

If you believe that this document breaches copyright please contact us providing details, and we will remove access to the work immediately and investigate your claim. 


\title{
Scaling Up Cooperative Game Theory Based Energy Management using Prosumer Clustering
}

\author{
Liyang Han, Student Member, IEEE, Thomas Morstyn, Member, IEEE, \\ and Malcolm D. McCulloch, Senior Member, IEEE
}

\begin{abstract}
Game theory based energy sharing schemes emerged in recent years to incentivize efficient management of the increasing amount of distributed energy resources. Among these, cooperative game theoretic schemes provide detailed financial incentives on the individual prosumer level. The nucleolus, a mechanism to allocate these financial incentives, has been proven to guarantee the prosumers' willingness to participate. However, the computation time of the nucleolus increases exponentially with the number of participants, strictly limiting the size of this scheme. This study proposes to incorporate clustering techniques to estimate the nucleolus at reduced computation times, where a novel marginal contribution profile is used as the clustering features. A stratified random sampling based approach is formulated to evaluate the estimation performance, showing that the proposed method is able to scale up the cooperative energy management scheme from less than 15 players to over 100 players while maintaining high accuracy of the nucleolus estimation.
\end{abstract}

Index Terms-Prosumers, energy management, cooperative game theory, nucleolus, clustering, sampling, energy storage

\section{NOMENCLATURE}

$i \quad$ Prosumer index

j Cluster index.

$m \quad$ Stratum sample size for estimation evaluation.

$t \quad$ Timestep.

$\alpha \quad$ Mixing probability in a Gaussian Mixture Model.

$\delta \quad$ Stratified sampling convergence threshold for evaluating nucleolus estimation.

$\Delta \mathbf{F} \quad$ Grand coalition marginal contribution profile.

$\varepsilon \quad$ Excess of a coalition.

$\eta^{i n} \quad$ Charge efficiency of energy storage units.

$\eta^{\text {out }}$ Discharge efficiency of energy storage units.

$\boldsymbol{\theta}$ Components in a Gaussian Mixture Model.

$\boldsymbol{\mu} \quad$ Mean vector of a Gaussian Distribution.

$\nu \quad$ Nucleolus payoff allocation.

$\pi(\mathcal{N}) \quad$ Set of all possible permutations with $\mathcal{N}$.

$\Sigma \quad$ Covariance Matrix of a Gaussian Distribution. $b \quad$ Charge (positive) or discharge (negative) energy of energy storage units.

$\underline{b} \quad$ Discharge limit of energy storage units.

$\bar{b} \quad$ Charge limit of energy storage units.

c Centroid feature profile.

cl Cluster of prosumers.

$e \quad$ Energy capacity of energy storage units.

f Feature profile for clustering.

$F \quad$ Coalitional energy cost.

$g \quad$ Cluster assignment of prosumers.

$G^{R} \quad$ Minimized coalitional energy cost.

$K \quad$ Total number of clusters.

$M_{i l} \quad$ Set of permutation samples from $P_{i l}$ of size $m$.

$N \quad$ Number of prosumers in the grand coalition.

$\mathcal{N} \quad$ Prosumer grand coalition.

$O \quad$ A permutation of $\mathcal{N}$.

$P_{i l} \quad$ Set of all $O$ 's that assign player $i$ to position $l$.

Pre $e^{i} \quad$ Set of predecessors of the player $i$ in any $O$.

$q \quad$ Net energy consumption (positive) or generation (negative) without energy storage.

$R \quad$ Total number of timesteps.

$r^{e x} \quad$ Export price of energy.

$r^{i m} \quad$ Import price of energy.

$\mathfrak{R}_{m} \quad$ Combined set of all $M_{i l}, \forall i, l \in[1, N]$.

$\mathfrak{S}_{i} \quad$ Set of coalitions of clusters with each element being the joint coalition of $i$ and a coalition in $\mathbf{U}_{i}$.

$S o C^{0}$ Initial state of charge of energy storage units.

$\underline{S o C} \quad$ State of charge lower limit of energy storage units.

$\overline{\mathrm{SoC}}$ State of charge upper limit of energy storage units.

$\mathcal{T} \quad$ Prosumer coalition.

$\mathfrak{T}^{c l_{\mathcal{K}}} \quad$ Prosumer coalitions for nucleolus estimation.

$\mathfrak{T}^{\mathcal{N}} \quad$ Set of all the possible coalitions in an $N$-player cooperative energy management scheme.

$\mathcal{U} \quad$ Set of cluster indices for a coalition of clusters.

$\mathbf{U}_{i} \quad$ Set of coalitions of clusters that do not contain $i$.

$v \quad$ Value of a coalition.

$x \quad$ Prosumer payoff allocation.

\section{INTRODUCTION}

This work was supported in part by the Engineering and Physical Sciences Research Council under Grants EP/N03466X/1, EP/S000887/1, and EP/S031901/1, and in part by the Oxford Martin Programme on Integrating Renewable Energy.

Liyang Han has recently finished his $\mathrm{PhD}$ defense at the University of Oxford (July 2020), and is currently with the Department of Electrical Engineering, Technical University of Denmark, 2800 Lyngby, Denmark (email: liyha@elektro.dtu.dk).

Thomas Morstyn is with the School of Engineering, University of Edinburgh, Edinburgh EH9 3JL, U.K. (email: thomas.morstyn@ed.ac.uk).

Malcolm D. McCulloch is with the Department of Engineering Science, University of Oxford, Oxford OX1 2JD, U.K. 
identified as a useful tool to address this [4]. Energy prices are often structured to inform ES owners on how to schedule their ES units, and they can be adopted by individuals or shared on a community scale [5].

Prosumer-centric local energy markets emerged in recent years as an economic solution for coordinating flexible resources, such as ES, in the distribution network [6]. Under the common assumption that the price to export energy back to the grid is lower than the price to import [7], they create economic benefits for the prosumer participants by allowing them to trade or share energy within the local market.

Assuming prosumers are free to choose the market action that yields the best economic outcome for themselves, game theory, as a subject to study strategic situations, has been widely adopted as the theoretic framework for these prosumercentric markets.

Non-cooperative game theory based schemes rely on dynamic pricing of energy [8]. The iterative communication between the electricity suppliers and the end users converges to the real-time prices, or the Nash equilibrium [9], from which no player can be financially better off deviating. However, noncooperative games lack protection against market manipulation [10], and they do not consider alternative, and potentially economically preferable, market solutions for the subsets of participants, causing concerns about market stability [11].

Cooperative game theory, on the other hand, focuses on the fairness and the level of incentive of the profit allocation [12]. A cooperative game theoretic scheme for local energy trading was first proposed in [13], offering prosumer participants stabilizing payoffs, meaning that no prosumer or coalition can be financially better off by leaving the grand coalition, which contains all the prosumers considered for the scheme. However, ES operation was not considered in this scheme. Considering multi-period time-of-use prices for both importing and exporting energy, [14] constructs a cooperative energy management scheme allowing ES units to actively participate in the market. It was proven in [11] that the nucleolus, a payoff allocation that minimizes the dissatisfaction of coalitions, is stabilizing.

However, as the number of prosumers in this cooperative energy management scheme increases, the nucleolus becomes computationally intractable, because 1) it requires solving convex optimization problems to calculate the values of all coalitions, the number of which is an exponential function of the number of prosumers [15]; 2) given the values of all coalitions, the number of iterative steps required to compute the nucleolus is also an exponential function of the number of prosumers [16]. A stabilizing payoff allocation in an analytical expression was proposed in [17], and a worst-case excess method was used in [18] to compute a partial nucleolus. However, both of these payoff allocations only address the second computational problem, leaving the coalition value calculations still intractable for large games. Among all the case studies for the cooperative energy management scheme in [11], [17], [18], the highest number of players shown is 15.

In the energy sector, clustering is often used to identify typical load patterns for energy pricing structure design [19] and electric load forecasting and management [20].
Considering the payoffs derived from the nucleolus of the cooperative energy management scheme heavily depend on the participants' consumption and generation profile patterns, clustering is therefore a promising technique to help estimate the nucleolus and overcome its computational challenges.

To speed up the nucleolus computation, the authors' previous work [21] uses K-means clustering to group prosumers into a reduced number of players, named clustered players. Instead of computing the nucleolus for individual prosumers, the nucleolus for these clustered players is computed, which is then divided within the clustered players. However, for a lack of means to distinguish the contributions of individual prosumers within each clustered player, the in-cluster payoff division is arbitrary, which results in individual payoffs deviating significantly from the actual nucleolus.

To scale up the cooperative energy management scheme while maintaining a high accuracy of nucleolus estimation, this study proposes a new clustering based method, which directly estimates the nucleolus for individual prosumers. For categorizing energy load patterns, K-means clustering is widely used as a reliable baseline clustering technique [22], but hierarchical clustering and Gaussian mixture model (GMM) are shown to have superior performances measured by the Clustering Validity Indexes (CVIs) according to [23]. $\mathrm{K}$-means, hierarchical, and GMM are chosen in this study to demonstrate the effectiveness of the proposed method, but the method can incorporate a wide range of clustering algorithms.

It is recognized that the CVIs, albeit standard metrics to evaluate clustering techniques, cannot measure the estimation accuracy of the proposed method. Knowing that the nucleolus is a stabilizing payoff allocation, this study proposes the novel use of stratified random sampling [24] to evaluate the estimation performance by testing the stability that the estimated nucleolus introduces to the market scheme.

The novel contributions of this study include:

- a clustering based method to estimate the nucleolus of all prosumer participants in the cooperative energy management scheme. This method can incorporate a range of clustering techniques, and significantly improves the scalability of the cooperative energy management scheme proposed in [11], [17], [18].

- the formulation of the grand coalition marginal contribution profile as the clustering feature vector. It significantly improves the nucleolus estimation accuracy by capturing effectively prosumers' individual traits that are closely linked to their nucleolus payoffs.

- a performance evaluation approach based on a stratified random sampling technique. It is used to compare the estimation performances of different clustering techniques coupled with different clustering features.

\section{The Cooperative Game Theory Based Energy MANAGEMENT SCHEME}

Prosumers are defined as proactive consumers who actively control their energy behaviors in [2]. This study takes on a broader definition and considers a prosumer as any energy consumer who is financially incentivized to participate in a 
local energy management scheme. Cooperative game theory offers a framework to coordinate the energy operation of prosumers within a distribution network while offering them financial rewards for participation.

The cooperative game theory based energy management schemes proposed in [11], [17], [18] all define the value of forming coalitions as the energy cost savings from joint prosumer energy management within those coalitions. Overall, these prosumer energy management schemes follow three key steps: Step 1 is to establish the objective of cooperation, which is to optimally schedule the ES units and flexible loads to minimize the joint energy cost; Step 2 is to quantify the value of forming each coalition by calculating the energy cost savings from cooperative energy management; Step 3 is to calculate the individual payoffs by allocating the grand coalition energy cost savings to the participating prosumers. These three steps are detailed in the following subsections respectively. The presented framework is representative of, and can be adapted to, a range of cooperative game theoretic schemes applied in joint energy management.

\section{A. Coalitional Energy Cost}

In an $N$-prosumer cooperative energy management scheme, the set of all $N$ prosumers is defined as the grand coalition $\mathcal{N}=\{1,2, \ldots, N\}$. Each prosumer is indexed by $i: i \in \mathcal{N}$. Any subset of the grand coalition $\mathcal{T}: \mathcal{T} \subseteq \mathcal{N}$ is called a prosumer coalition. Note that a set of a single player $\{i\}$ is also considered a coalition. Given $R$ timesteps $(t=1,2, \ldots, R)$ with a time interval of $\Delta t$, the coalitional energy cost for $\mathcal{T}$ at timestep $t$ is written as

$$
F_{t}^{\mathcal{T}}(\mathbf{b})=\sum_{i \in \mathcal{T}}\left\{r_{t}^{i m}\left[q_{i t}+b_{i t}\right]^{+}+r_{t}^{e x}\left[q_{i t}+b_{i t}\right]^{-}\right\}
$$

where $[z]^{+}=\max (z, 0),[z]^{-}=\min (z, 0)$, and subscripts $i$ and $t$ are indices for the players and the timesteps. The electricity import price $r_{t}^{i m}(£ / k W h)$ and export price $r_{t}^{e x}$ $(£ / k W h)$ are known parameters, as well as $q_{i t}(k W h)$, which denotes the net energy consumption (positive) or generation (negative) over $\Delta t$ without ES. The variables are ES charge (positive) or discharge (negative) energy amounts $(\mathrm{kWh})$ over $\Delta t$, denoted by $\mathbf{b}=\left[b_{i t}\right], \forall i \in[1, N], \forall t \in[1, R]$.

With the increasing penetration of distributed generation, the price to export energy has largely fallen below the price to import energy [7]. With the assumption that both the electricity import and export price structures can vary with time as long as $r_{t}^{i m}>r_{t}^{e x}, \forall t$, the operation of ES units within coalition $\mathcal{T}$ can be scheduled to minimize the coalitional energy cost:

$$
\begin{aligned}
& G^{R}(\mathcal{T})= \min _{\mathbf{b}} \sum_{t=1}^{R} F_{t}^{\mathcal{T}}(\mathbf{b}) \\
& \text { s.t. } \quad \underline{b}_{i} \leq b_{i t} \leq \bar{b}_{i}, \quad \forall i \in \mathcal{T}, \forall t \in[1, R] \\
& e_{i} \underline{S o C}_{i} \leq e_{i} S o C_{i}^{0}+\sum_{t=1}^{r}\left(\left[b_{i t}\right]^{+} \eta_{i}^{\text {in }}+\left[b_{i t}\right]^{-} / \eta_{i}^{\text {out }}\right) \\
& \leq e_{i} \overline{S o C}_{i}, \quad \forall i \in \mathcal{T}, \forall r \in[1, R] \\
& \sum_{t=1}^{R}\left(\left[b_{i t}\right]^{+} \eta_{i}^{i n}+\left[b_{i t}\right]^{-} / \eta_{i}^{\text {out }}\right)=0, \quad \forall i \in \mathcal{T}
\end{aligned}
$$

(a) Indiv. Consumptions

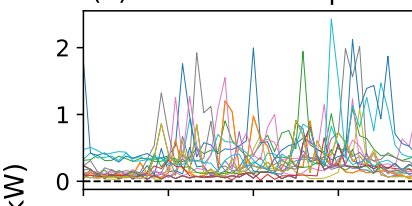

(c) Joint Net Load and ES Op. (b) Indiv. Net Loads

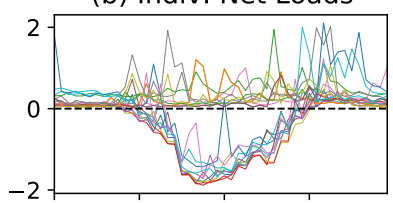

(d) Joint Load inc. ES

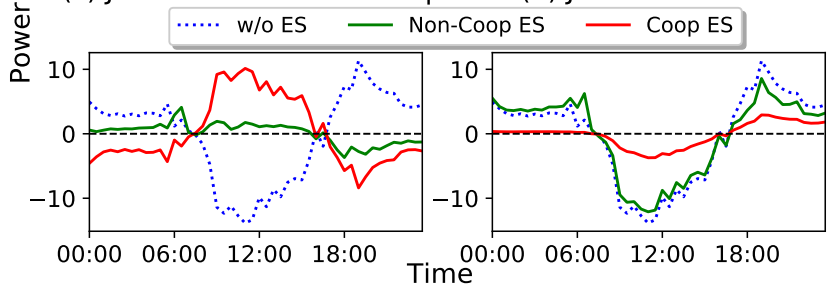

Fig. 1: 20 Prosumer loads: (a) individual load consumptions; (b) individual consumptions + PV generations; (c) blue dotted: joint consumption + PV generation, green: joint non-cooperative ES operation, red: joint cooperative ES operation; (d) blue dotted: joint consumption + PV generation, green: joint consumption + PV generation + non-cooperative ES operation, red: joint consumption + $\mathrm{PV}$ generation + cooperative ES operation.

where (3), (4), and (5) set the ES power constraints, energy constraints, and cycle constraints ${ }^{1}$ respectively. Each prosumer $i$ 's ES system has an energy capacity $(k W h)$ of $e_{i} \geq 0$, a charge limit $(k W h)$ of $\bar{b}_{i} \geq 0$ and a discharge limit $(k W h)$ of $\underline{b}_{i} \leq 0$ over the time span of $\Delta t$, a charge efficiency of $\eta_{i}^{\text {in }} \in(0,1)$ and a discharge efficiency of $\eta_{i}^{\text {out }} \in(0,1)$, an upper state of charge limit of $\overline{S o C}_{i} \in[0,1]$ and a lower state of charge limit of $\underline{S o C}_{i} \in[0,1]$, and an initial state of charge of $S o C_{i}^{0} \in[0,1]$. For a prosumer without ES, their energy capacity and charge/discharge limits are all set to zeros.

A 20-prosumer cooperative energy management example is demonstrated in Fig. 1. The model inputs can be found in Section V. It shows that in order to minimize the coalition energy cost, the cooperative scheme tries to match consumption and generation, which results in a flatter cooperative load profile. The optimal scheduling of energy operation is based on the predicted consumption and generation, and the impact of the prediction uncertainties on the outcome of the model is discussed in Case Study V-C.

The cooperative energy management scheme coordinates resources within a local distribution network, which is not expected to have significant losses and constraints between prosumers. Additionally, due to the load flattening effect of cooperation presented in Fig. 1, it is expected to reduce losses and alleviate upstream congestion. The cooperative energy management scheme focuses on the financial benefits for the prosumers, which do not consider losses and network constraints at this stage, but it would be an important area for future work if the scheme was scaled across larger networks.

\section{B. Value of Coalitions}

Cooperative game theory is a framework for allocating the profit from cooperation to the participants. It consists two main

\footnotetext{
${ }^{1}$ ES units are restored to their original states of charge at the end of each simulation period to ensure that ES units are only a resource for arbitrage instead of generation.
} 
tasks: 1) quantification of the value of cooperation within coalitions; 2) allocation of the total profit achieved through the grand coalition cooperation to all the participants. In a cooperative energy management scheme, the value function of a coalition is defined as the energy cost savings as a result of forming the coalition:

$$
v(\mathcal{T})=\sum_{i \in \mathcal{T}} G^{R}(\{i\})-G^{R}(\mathcal{T})
$$

where $\sum_{i \in \mathcal{T}} G^{R}(\{i\})$ is the sum of the energy costs of all the prosumers within coalition $\mathcal{T}$ by having them schedule their DER operations to minimize their own costs, and $G^{R}(\mathcal{T})$ is the minimized coalitional energy cost achieved by having all prosumers within coalition $\mathcal{T}$ schedule their DER operations cooperatively. Using this definition, the value of the grand coalition $v(\mathcal{N})$ is the total energy cost savings of a cooperative energy management scheme, which determines the total amount of payoffs that can be allocated to the participants.

\section{Payoff Allocation and Nucleolus}

Having obtained the total amount of payoffs, the next step is to determine how to allocate it to each prosumer.

Vector $\mathbf{x}$ is denoted as the payoff allocation, and its entry $x_{i}$ represents the payment to prosumer $i \in \mathcal{N}$. Payoffs can be allocated in various ways, but in order to attract participation, a payoff allocation needs to be minimally

1) Efficient: $\sum_{i \in \mathcal{N}} x_{i}=v(\mathcal{N})$. This requires the entirety of the profit created by the grand coalition to be allocated to the prosumers.

2) Individually Rational: $x_{i} \geq v(\{i\}), \forall i \in \mathcal{N}$. This ensures that no prosumer is financially worse off.

However, even an efficient and individually rational payoff allocation does not necessarily guarantee everyone being satisfied in the grand coalition, as some players may be able to achieve higher payoffs by forming smaller coalitions.

Definition 1 (Excess): An energy coalition's dissatisfaction with respect to $\mathbf{x}$ is measured by its excess defined as

$$
\varepsilon(\mathbf{x}, \mathcal{T})=v(\mathcal{T})-\sum_{i \in \mathcal{T}} x_{i}
$$

If $\varepsilon(\mathbf{x}, \mathcal{T})>0$, it means the value of coalition $\mathcal{T}$ is higher than the sum of the profits allocated to the prosumers within $\mathcal{T}$ under the grand coalition payoff scheme $\mathbf{x}$. Since $v(\mathcal{T})$ represents the energy cost savings that can be achieved for coalition $\mathcal{T}$ alone by having its members cooperatively schedule their DER operations, it signals that $\mathcal{T}$ is better off on its own and can offer higher payoffs to its members than the grand coalition. Reversedly, if none of the excesses are positive, there is no incentive for any coalition to defect from the grand coalition. Therefore, the excess is an indicator of whether a payoff allocation is stabilizing, or can guarantee the stability of the cooperative energy management scheme.

Definition 2 (Stabilizing): Given a payoff allocation $\mathbf{x}$, it is said to be stabilizing if $\varepsilon(\mathbf{x}, \mathcal{T}) \leq 0, \forall \mathcal{T} \subseteq \mathcal{N}$.

The set of stabilizing payoff allocations for a cooperative game is called the core. Two popular payoff allocations, the
Shapley value [25] and the nucleolus, are analyzed and compared for the cooperative energy management scheme in [11], which proves that the Shapley value is not in the core, while the nucleolus is. In fact, the nucleolus is the only known payoff allocation that actively validates the coalition dissatisfaction, for its computation lexicographically minimizes excesses of all the possible coalitions [26]. Therefore, this study only discusses the computation of the nucleolus.

Algorithm 1 explains the iterative steps of lexicographically minimizing the excesses of a given set of coalitions. In order to compute the nucleolus $\boldsymbol{\nu}$, the set of all the possible coalitions in an $N$-player cooperative energy management scheme is denoted as $\mathfrak{T}^{\mathcal{N}}:=\{\mathcal{T} \mid \mathcal{T} \subseteq \mathcal{N}\}$. By setting $\mathfrak{T}^{*}=\mathfrak{T}^{\mathcal{N}}$, Algorithm 1 outputs $\mathrm{x}^{*}$, and $\boldsymbol{\nu}=\mathrm{x}^{*}$.

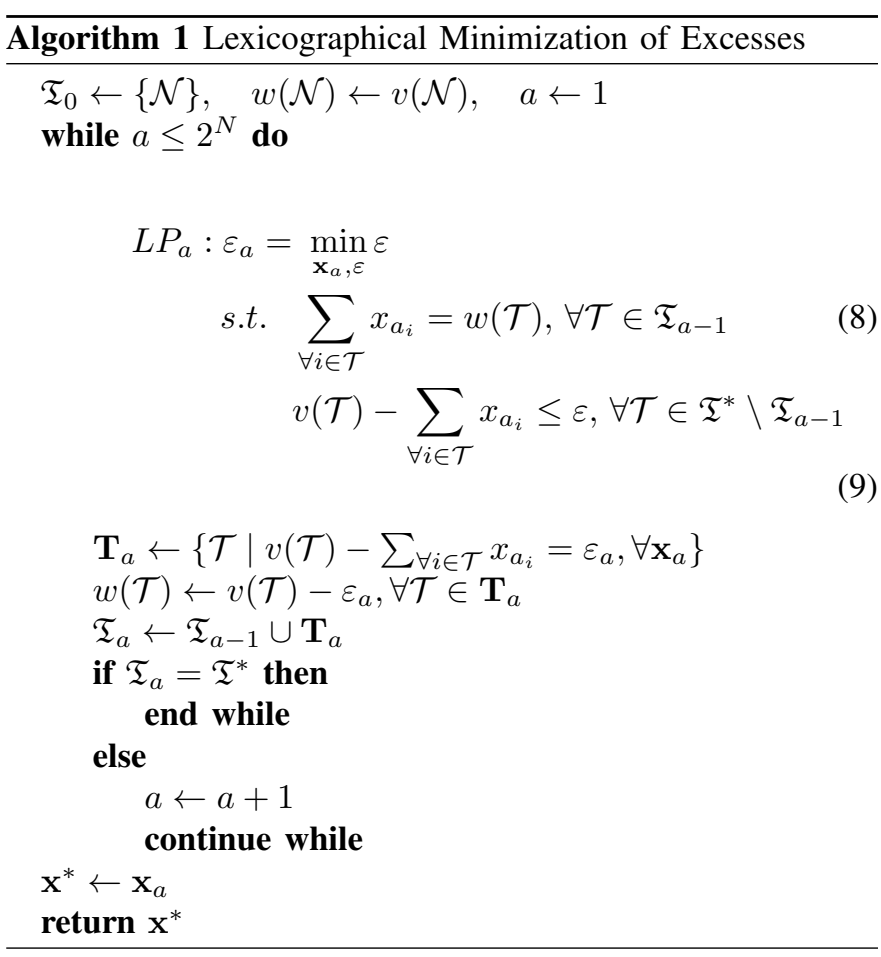

Here, subscript $a$ numbers each linear program $(L P)$ in the iteration process. Constraints (8) ensure the efficiency criterion of the payoff allocation, and fix the excesses of the coalitions that have been minimized in all previous iterations. Constraints (9) cap the excesses for all remaining coalitions at $\varepsilon$ that is then minimized in $L P_{a}$. At the end of each iteration, all the binding constraints among (9) are identified, with their corresponding coalitions defined as $\mathbf{T}_{a} . \mathbf{T}_{a}$ 's excesses are then fixed for all subsequent iterations using (8).

\section{Computational Complexity}

The cooperative energy management scheme offers a way to encourage DER collaboration while ensuring that all prosumers are financially incentivized to remain in the grand coalition. Its computation involves obtaining the grand coalition profit $v(\mathcal{N})$ and allocating the profit to the participating prosumers based on the nucleolus. The grand coalition profit is calculated by solving cost minimization problems for the grand coalition and all $N$ prosumers using (2). The actual 
implementation of the grand coalition cooperation can be through centralized or distributed control algorithms [27], [28], but is outside of the scope of this study.

The problems needed for calculating the grand coalition profit only make up a small portion of the nucleolus computation, whose complexity is two-folded. First, the values need to be calculated for all possible coalitions, the number of which is $\left|\mathfrak{T}^{\mathcal{N}}\right|=2^{N}-1$. Second, in solving for the lexicographically minimal excesses of all coalitions, both the number of linear problems and the number of constraints in each linear problem are $\sim \mathcal{O}\left(2^{N}\right)$. Both are closely linked to the number of coalitions, so the key to reducing the computation time is to limit the number of coalitions considered in the computation.

The nucleolus payoff each player receives is determined by their contribution to the coalitions, which means players of similar energy behaviors tend to receive similar payoffs. Meanwhile, the value of cooperation relies on the matching of generation and consumption within the coalition, which means it is the heterogeneity within a coalition that creates value for its members. By capturing the similarities and differences among players, a reduced number of coalitions that are most likely to be dissatisfied can be identified. It then becomes possible to estimate the nucleolus by only minimizing the excesses of these coalitions. For this purpose, Section III investigates the use of different clustering techniques to identify similarities and differences, and Section IV explores the use of these clustering techniques for estimating the nucleolus.

\section{Clustering TeChniques for Classifying LOAD PROFILES}

Clustering algorithms for identifying load patterns have been studied extensively in the literature [19], [20], [22], [23], [29]. K-means clustering is often used as a baseline clustering technique [22], [29], and a recent comparative study identifies hierarchical clustering with ward linkage and Gaussian Mixture Model (GMM) with a full-unshared covariance matrix as the best performing clustering techniques in classifying load profiles [23]. These three clustering techniques are used to validate the performance of the proposed clustering based method for nucleolus estimation, which is detailed in Section IV. The purpose of choosing a selection of popular clustering techniques is to demonstrate the effectiveness of the proposed method; a comprehensive investigation of the best possible clustering technique to use is left for future work.

For clarity, prosumers are grouped into $K$ clusters, denoted as $c l_{\mathcal{K}}:=\left\{c l_{1}, c l_{2}, \ldots, c l_{K}\right\}$, and the clustering assignment is $\boldsymbol{g}: g_{i}=j \mid i \in c l_{j}, \forall i \in \mathcal{N}$. Here, $K$ is applicable for not only K-means, but all clustering techniques.

\section{A. K-Means Clustering}

K-means clustering is a widely used data clustering technique for unsupervised learning tasks. It requires a predetermined number of clusters $K$, and an initial set of $K$ centroids usually chosen at random among the given instances. Clustering is done through an iterative process of minimizing the sum of all the squared errors between all instances and their corresponding centroids. To cluster prosumers, the error is measured by the Euclidean distance. The Euclidean distance between Player $i$ and Cluster $c l_{j}$ 's centroid is defined as

$$
d\left(\mathbf{f}_{i}, \mathbf{c}_{j}\right)=\sqrt{\sum_{t}^{R}\left(f_{i t}-c_{j t}\right)^{2}}
$$

where $\mathbf{f}_{i}$ denotes player $i$ 's feature profile, and $\mathbf{c}_{j}$ denotes cluster $c l_{j}$ 's centroid feature profile.

Each K-means iteration completes two tasks:

1) Assign each instance to the cluster with the closest centroid profile:

$$
g_{i} \leftarrow \underset{j}{\arg \min } d\left(\mathbf{f}_{i}, \mathbf{c}_{j}\right)
$$

2) Recompute the centroid profiles for each cluster based on the instances assigned to it using:

$$
\mathbf{c}_{j}=\underset{\mathbf{c}}{\arg \min } \sum_{i \in c l_{j}} d^{2}\left(\mathbf{f}_{i}, \mathbf{c}\right)=\frac{\sum_{i \in c l_{j}} \mathbf{f}_{i}}{\left|c l_{j}\right|}
$$

where $\left|c l_{j}\right|$ is the number of prosumers in Cluster $c l_{j}$.

These two tasks are repeated until the convergence criteria are met, which either means no changes in cluster assignment are observed or the number of iterations reaches the prespecified limit. Assuming the maximum number of iterations is set to iter $_{\max }$, the iterative steps of $\mathrm{K}$-means clustering for classifying feature profiles are detailed in Algorithm 2.

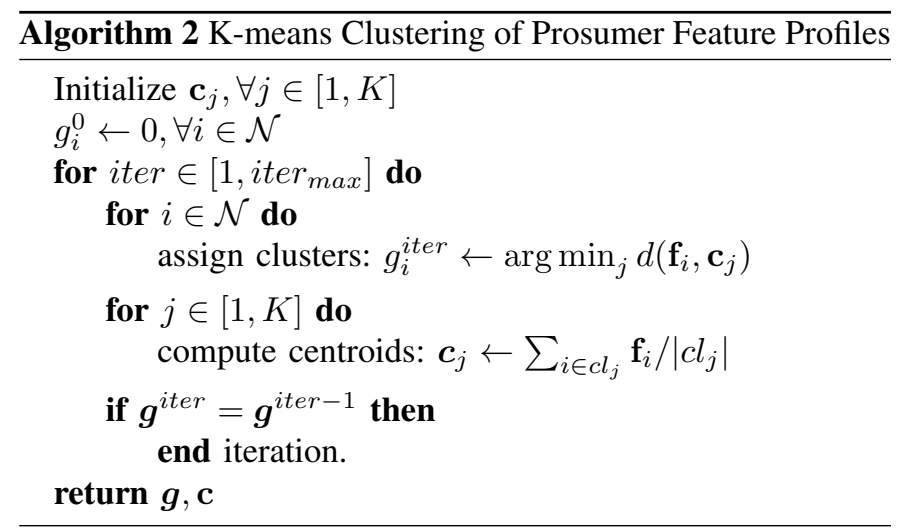

The random initial centroid selection coupled with this iteration process often traps the solution in the local minima, so it is advised to perform K-means repeatedly with different randomized initializations and pick the run with the lowest K-means objective function value denoted by $\min \sum_{i \in \mathcal{N}} d^{2}\left(\mathbf{f}_{i}, \mathbf{c}_{g_{i}}\right)$. In this study, K-means clustering is run 1000 times with different initial centroids. Fig. 2 shows an example of 100 prosumers grouped into 6 clusters based on their grand coalition cooperative energy profiles.

\section{B. Hierarchical Clustering}

Unlike K-means clustering, hierarchical clustering can be performed deterministically without a preset total number of clusters [30]. It is done by either an agglomerative (bottom-up) or a divisive (top-down) approach. Here, only the agglomerative approach is investigated as it is preferred in power system applications [23]. It begins by treating each instance as 


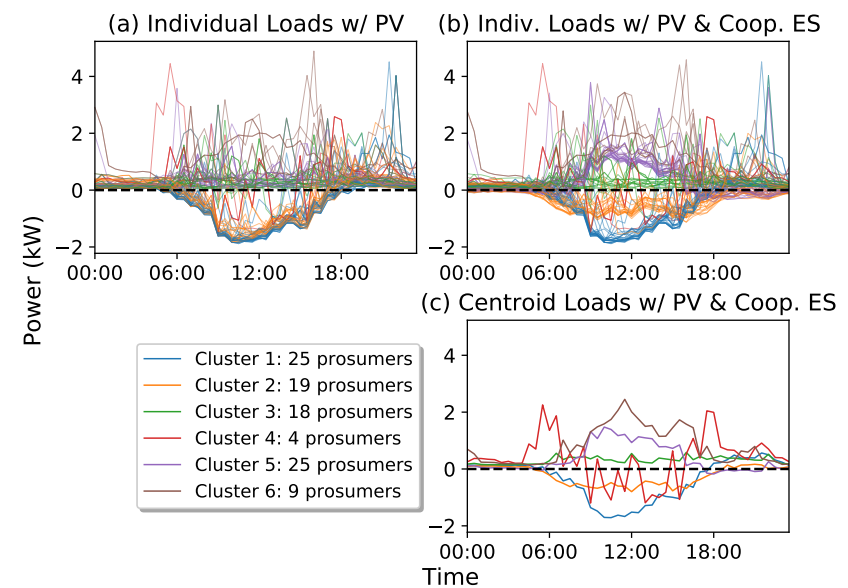

Fig. 2: Load profiles of 100 prosumers grouped into 6 clusters based on the grand coalition cooperative energy profiles: (a) individual consumptions + PV generations; (b) individual cooperative profiles: consumptions + PV + ES optimized for the grand coalition; (c) 6 centroid cooperative energy profiles using K-means clustering.

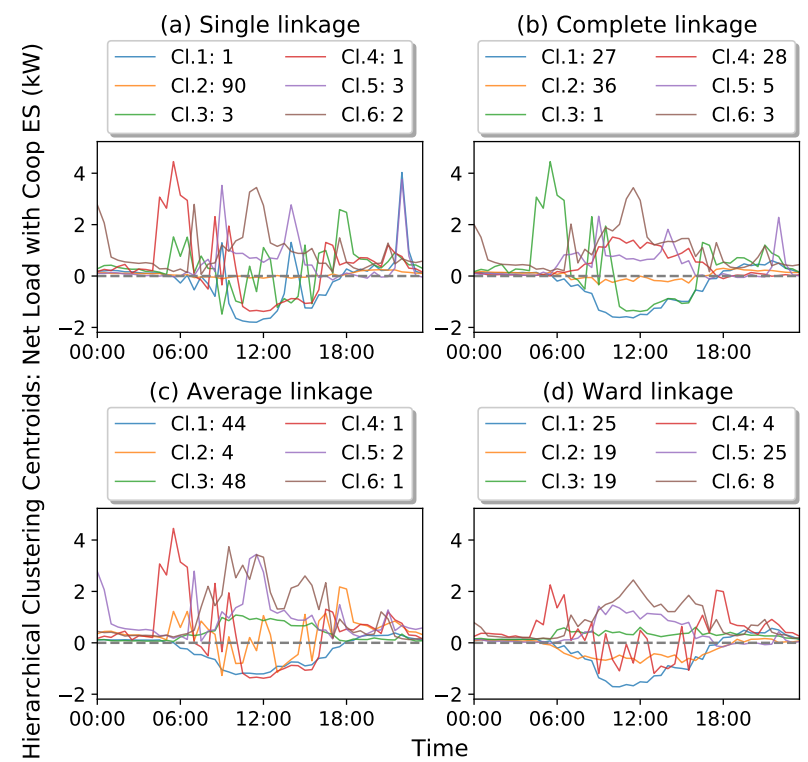

Fig. 3: Hierarchical clustering centroids of 100 prosumers' grand coalition cooperative energy profiles with different linkage criteria.

an individual cluster, and proceeds by continuously merging clusters that are most similar to each other. The Euclidean distance, defined in (10), is used to measure the dissimilarity between clusters [31].

A linkage criterion needs to be selected in order to perform hierarchical clustering. Based on the definition of similarity between clusters, there are four types of linkage criteria, namely single, complete, average and ward. Single linkage and complete linkage define the similarity between two clusters as the distance between the closest and farthest members respectively from the two clusters. Average linkage sets the similarity of two clusters as the average distance between all pairs of instances between the two. The ward criterion minimizes the increase in the sum of squared distances between all members of the clusters and their corresponding cluster centroids.
Using the same inputs as Fig. 2, Fig. 3 shows the results of sorting 100 prosumers into 6 groups using hierarchical clustering. As the process of hierarchical clustering does not involve the computation of centroids, all the centroid profiles are computed post clustering using $\mathbf{c}_{j}=\arg \min _{\mathbf{c}} \sum_{i \in c l_{j}} d^{2}\left(\mathbf{f}_{i}, \mathbf{c}\right)$. The main goal of clustering in this study is to identify both similarities and dissimilarities among all prosumers; hence sensitivity to outliers is undesirable. In the rest of this study only the ward linkage criterion is considered, for it produces the most even spread of prosumers among clusters. This observation is consistent with the results in [23].

\section{Gaussian Mixture Model (GMM)}

Both K-means clustering and hierarchical clustering are distance-based clustering techniques. GMM, on the other hand, is a probabilistic technique. It is part of the finite mixture models family, which develops a probability distribution function (PDF) that is a mixture of $K$ components: $\boldsymbol{\theta}=\left\{\boldsymbol{\theta}_{1}, \ldots, \boldsymbol{\theta}_{K}\right\}$. This PDF has the maximum likelihood to fit the given instances, given by

$$
p\left(\mathbf{f}_{i} \mid \boldsymbol{\theta}\right)=\sum_{j=1}^{K} \alpha_{j} p\left(\mathbf{f}_{i} \mid \boldsymbol{\theta}_{j}\right)
$$

where $\boldsymbol{\theta}_{j}=\left\{\theta_{j t} \mid t \in[1, R]\right\}$, and $\alpha_{j}$ is the mixing probability or weight of the $j$ th component $\boldsymbol{\theta}_{j}, \alpha_{j} \geq 0, j=1,2, \ldots, K$, and $\sum_{j=1}^{K} \alpha_{j}=1$. In the case of GMM, $\boldsymbol{\theta}_{j}=\left(\boldsymbol{\mu}_{j}, \boldsymbol{\Sigma}_{j}\right)$, where $\boldsymbol{\mu}_{j}$ is the $R$-dimensional mean vector, and $\boldsymbol{\Sigma}_{j}$ is the covariance matrix. (13) can be rewritten as

$$
p\left(\mathbf{f}_{i} \mid \boldsymbol{\theta}\right)=\sum_{j=1}^{K} \alpha_{j} \frac{\exp \left\{-\frac{1}{2}\left(\mathbf{f}_{i}-\boldsymbol{\mu}_{j}\right)^{\boldsymbol{\top}} \boldsymbol{\Sigma}_{j}^{-1}\left(\mathbf{f}_{i}-\boldsymbol{\mu}_{j}\right)\right\}}{(2 \pi)^{\frac{R}{2}}\left|\boldsymbol{\Sigma}_{j}\right|^{\frac{1}{2}}}
$$

The maximum likelihood estimate of $\{\boldsymbol{\mu}, \boldsymbol{\Sigma}, \boldsymbol{\alpha}\}$ is often obtained through an iterative expectation-maximization (EM) algorithm [29], [32]. To prevent convergence to local optima, GMM can be run repeatedly with different initial conditions [33]. In this study, the number of runs for GMM is set to be 100. Finally, clustering is completed by assigning each instance to the component with the highest posterior probability:

$$
p\left(k \mid \mathbf{f}_{i}\right)=\frac{\alpha_{k} p\left(\mathbf{f}_{i} \mid \boldsymbol{\theta}_{k}\right)}{\sum_{j=1}^{K} \alpha_{j} p\left(\mathbf{f}_{i} \mid \boldsymbol{\theta}_{j}\right)}, \quad k=1, \ldots, K
$$

To implement GMM, the structure of the covariance matrix needs to be specified. For each component, a full covariance matrix has uncorrelated dimensions, while a diagonal covariance matrix has correlated dimensions. A spherical covariance matrix is a diagonal covariance matrix that has the same covariance across all dimensions. Full, diagonal, spherical covariance matrices are unshared among components. Tied covariance matrices have uncorrelated dimensions, and are shared by all components.

Again, with the same inputs as Fig. 2 and Fig. 3, GMM is used to cluster 100 prosumers into 6 groups and the results are postprocessed to generate the centroid profiles, shown in Fig. 4. The most even spread of prosumers among clusters can be achieved with a full covariance matrix. For the reason explained in Section III-B, only the full covariance matrix, 


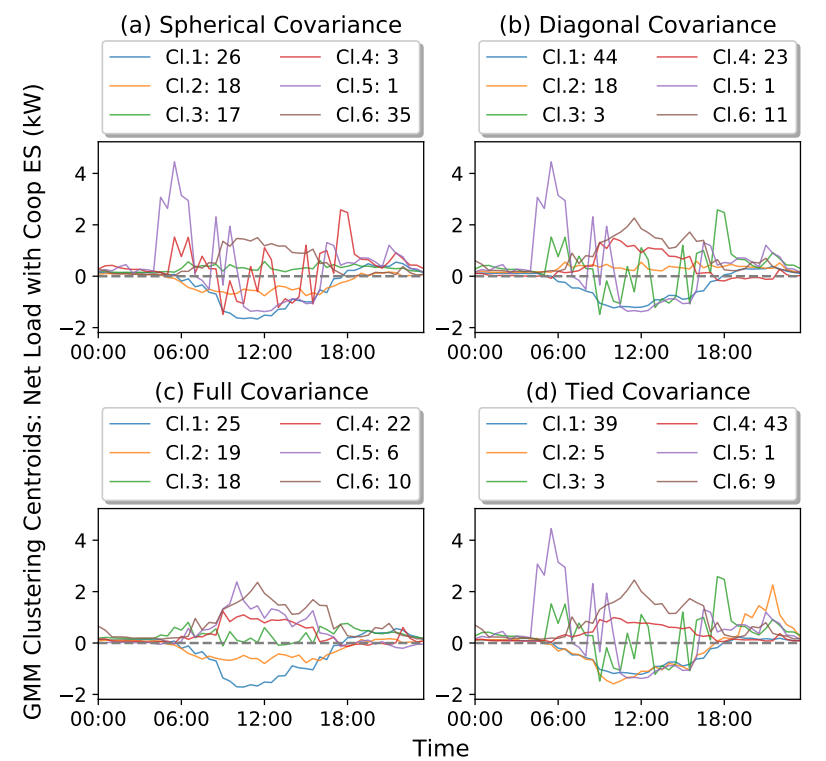

Fig. 4: GMM clustering centroids of 100 prosumers' grand coalition cooperative energy profiles with different covariance matrices.

which has uncorrelated dimensions, is considered for GMM clustering in the rest of this study.

\section{Nucleolus Estimation and Validation}

Coalitions with the most diverse mix of players tend to create the highest values for their members when independent from the grand coalition, and therefore they are most likely to defect. Reversely, a payoff allocation that prevents these types of coalitions from defecting is likely to retain other coalitions in the game as well. This is reflected in the algorithm for nucleolus computation, which is to iteratively minimize the excesses of all coalitions. Partially following this algorithm, the nucleolus can therefore be estimated by minimizing the excesses of selected coalitions of the highest diversity that are most likely to defect. Clustering, as described in the previous section, can identify dissimilarities among prosumers, offering a strategy to construct these coalitions of diverse populations. In this section, a nucleolus estimation algorithm is proposed incorporating clustering of prosumers with different features.

\section{A. Clustering for Nucleolus Estimation}

Both a coalition and a cluster represent a collective of players. However, while a prosumer coalition is any subset of the grand coalition, a prosumer cluster is a group of prosumers with similar traits sorted together with a clustering technique. Coalitions can adopt cooperative energy management and allocate profits to the participating prosumers, whereas clusters are created in this study only to facilitate the computation of the payoff allocation. Clustering has no influence on the optimal scheduling of the energy operation within coalitions.

The nucleolus estimation method presented in [21] defines a prosumer cluster as a clustered player, and simplifies the cooperative energy management scheme to a cooperative game among the clustered players. With $K$ clustered players
( $K \ll N)$, the computational complexity can be reduced from $\sim \mathcal{O}\left(2^{N}\right)$ to $\sim \mathcal{O}\left(2^{K}\right)$. However, the contributions of individual prosumers cannot be distinguished within each clustered player, leading to arbitrary in-cluster division of payoffs. As a result, the payoff allocations to the prosumers are highly inaccurate estimates of the nucleolus. A comparison of the estimation performances of this clustered players method and the proposed method is shown in Case Study V-A.

Clustering separates dissimilar players, but it does not provide any information about the differences between prosumers within the same cluster. To solve this issue, instead of treating each cluster as a distinctive player, every prosumer can be paired with clusters they do not belong to in order to form coalition samples for excess minimization. This way, not only is the diversity within the coalition samples ensured, but each prosumer's individuality is preserved as well. The proposed method for estimating the nucleolus is as follows:

1) Based on a certain prosumer feature (see Section IV-B), prosumers are separated into $K$ prosumer clusters, the complete set of which is denoted as $c l_{\mathcal{K}}:=\left\{c l_{j} \mid j \in \mathcal{K}\right\}$ where $\mathcal{K}=\{1,2, \ldots, K\}$. The clustering assignment is $\boldsymbol{g}: g_{i}=j \mid i \in c l_{j}, \forall i \in \mathcal{N}$.

2) A coalition of prosumer clusters is defined as $c l_{\mathcal{U}}:=$ $\left\{c l_{j} \mid \forall j \in \mathcal{U}\right\}, c l_{\mathcal{U}} \subseteq c l_{\mathcal{K}}$. Any $c l_{\mathcal{U}}$ can be converted to a coalition of prosumers by using $Q\left(c_{\mathcal{U}}\right)=\left\{i \mid c l_{g_{i}} \in\right.$ $\left.c l_{\mathcal{U}}\right\}$. Therefore, all the prosumer coalitions derived from coalitions of prosumer clusters can be written as $\mathfrak{U}=$ $\left\{\mathcal{T} \mid \mathcal{T}=Q\left(c l_{\mathcal{U}}\right), \forall c l_{\mathcal{U}} \subseteq c l_{\mathcal{K}}\right\}$

3) For each prosumer $i \in \mathcal{N}$, the set of coalitions of prosumer clusters that do not contain $i$ is $\mathbf{U}_{i}=$ $\left\{c l_{\mathcal{U}} \mid \forall c l_{\mathcal{U}} \subseteq\left(c l_{\mathcal{K}} \backslash\left\{c l_{g_{i}}\right\}\right)\right\}$. If prosumer $i$ is then added to each of the coalitions in $\mathbf{U}_{i}$, this new set of prosumer coalitions becomes $\mathfrak{S}_{i}=\{\mathcal{T} \mid \mathcal{T}=$ $\left.Q\left(c l_{\mathcal{U}}\right) \cup\{i\}, \forall c l_{\mathcal{U}} \in \mathbf{U}_{i}\right\} . \mathfrak{S}_{i}$ is then constructed for every prosumer, all of which are combined into a single set $\mathfrak{S}=\left\{\mathcal{T} \mid \mathcal{T} \in \mathfrak{S}_{i}, \forall i \in \mathcal{N}\right\}$.

4) Finally, the set of prosumer coalition samples for excess minimization is constructed, denoted as $\mathfrak{T}^{c l_{\mathcal{K}}}=\mathfrak{S} \cup \mathfrak{U}$.

In addition to the coalitions of whole clusters denoted by $\mathfrak{U}$ (used as coalitions of clustered players in [21]), the coalition sample set $\mathfrak{T}^{c l_{\mathcal{K}}}$ also includes $\mathfrak{S}$ that groups every prosumer $i$ with all possible coalitions of prosumer clusters that do not contain $i$. This diversity based paring means $\mathfrak{S}_{i}$ very likely contains the coalition samples that can provide the highest payoffs to player $i$. Therefore, by minimizing the excesses of these coalitions, player $i$ 's tendency to defect from the grand coalition can be significantly reduced. The nucleolus estimation $\boldsymbol{\nu}^{\text {est }}$ can thus be obtained by implementing Algorithm 1 with $\mathfrak{T}^{*}=\mathfrak{T}^{c l_{\mathcal{K}}}$. The size of $\mathfrak{T}^{c l_{\mathcal{K}}}$ is bounded by

$$
\begin{aligned}
\left|\mathfrak{T}^{c l \mathcal{K}}\right| & \leq|\mathfrak{U}|+|\mathfrak{S}| \\
& \leq\left(2^{K}-1\right)+\left(2^{K-1} N\right) \\
& =2^{K-1}(N+2)-1
\end{aligned}
$$

Where the inequalities in (14) and (15) both occur when clustering yields one or more single-prosumer clusters. Therefore, given a selected number of clusters $K$, both the number of coalitions that require a value calculation and the number of 
iterations in the nucleolus calculation become $\sim \mathcal{O}(N)$, a significant reduction compared to the full nucleolus calculation $\sim \mathcal{O}\left(2^{N}\right)$ as explained in Section III.

\section{B. Clustering Features}

The choice of the clustering features has a direct impact on the clustering results. To identify typical load patterns, customer consumption profiles are used as the clustering features [34]. However, in a cooperative energy management scheme, some prosumers' energy profiles are not fixed but dependent on the coalition they are in. Therefore, the following clustering features suitable for identifying similarities among prosumers are investigated for cooperative energy management.

1) Grand coalition cooperative energy profile $\mathbf{p}:=$ $\left\{\mathbf{p}_{1}, \mathbf{p}_{2}, \ldots, \mathbf{p}_{N}\right\}$. Although prosumers' DER operation needs to be simulated under different coalition configurations to calculate these coalitions' values, the grand coalition yields the actual total profit. The cooperative energy profile of Prosumer $i$ may differ based on the coalition they participate in, but their grand coalition cooperative energy profile $\mathbf{p}_{i}$ is the most representative of its actual contribution to the scheme, capturing information about the prosumer's fixed energy consumption, PV generation, and ES operation. Based on (1),

$$
\begin{aligned}
\mathbf{b}^{\mathcal{N}} & =\underset{\mathbf{b}}{\arg \min } \sum_{t=1}^{R} F_{t}^{\mathcal{N}}(\mathbf{b}) \\
\mathbf{p}_{i} & =\mathbf{q}_{i}+\mathbf{b}_{i}^{\mathcal{N}}, \forall i \in \mathcal{N}
\end{aligned}
$$

Fig. 2 shows an example of using K-means to group 100 prosumers into 6 prosumer clusters based on their grand coalition cooperative energy profiles.

2) Grand coalition marginal contribution profile $\Delta \mathbf{F}:=$ $\left\{\Delta \mathbf{F}_{1}, \Delta \mathbf{F}_{2}, \ldots, \Delta \mathbf{F}_{N}\right\}$. In the cooperative energy management scheme, the value of a coalition corresponds to its energy cost savings, and the payoff is closely linked to a prosumer's marginal contributions to coalitions. Since the total profit is created through cooperation within the grand coalition, the marginal contribution to the grand coalition can be used as an identifier of the participating prosumers. Therefore, $\Delta \mathbf{F}$ is investigated as a clustering feature. Expanding the definition of the optimal ES operation in (16) to any coalition $\mathcal{T}$ :

$$
\mathbf{b}^{\mathcal{T}}=\underset{\mathbf{b}}{\arg \min } \sum_{t=1}^{R} F_{t}^{\mathcal{T}}(\mathbf{b})
$$

$\Delta \mathbf{F}_{i}$ is defined as the total cost savings by having Player $i$ join the existing cooperative scheme of $\mathcal{N} \backslash\{i\}$ :

$$
\Delta F_{i t}=F_{t}^{\mathcal{N} \backslash\{i\}}\left(\mathbf{b}^{\mathcal{N} \backslash\{i\}}\right)+F_{t}^{\{i\}}\left(\mathbf{b}^{\{i\}}\right)-F_{t}^{\mathcal{N}}\left(\mathbf{b}^{\mathcal{N}}\right)
$$

Fig. 5 shows an example of using GMM to group 100 prosumers into 6 prosumer clusters based on their grand coalition marginal contribution profiles.

Both the grand coalition cooperative energy profile and the grand coalition marginal contribution profile contain identifying information about the prosumers while they are participants in the grand coalition. The grand coalition cooperative
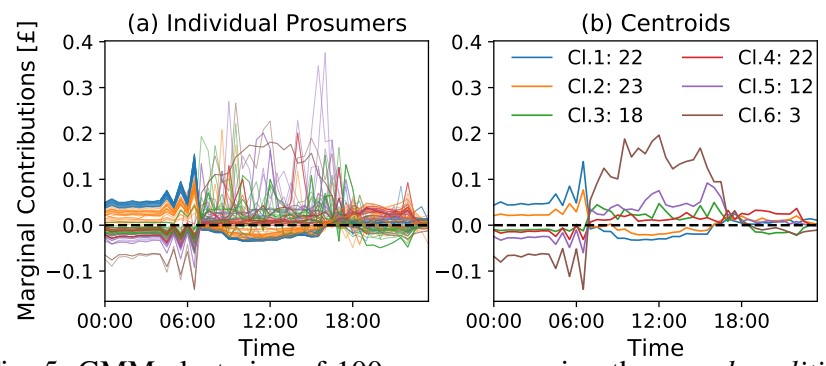

Fig. 5: GMM clustering of 100 prosumers using the grand coalition marginal contribution profiles as clustering features: (a) individual marginal contribution profiles; (b) centroid marginal contribution profiles with legend showing the number of prosumers in each cluster

energy profile is better at retaining information about the magnitude of a prosumer' energy consumption and generation, while the grand coalition marginal contribution profile is better at retaining information about the direct impact of a prosumer's energy operation on the total profit. Since clustering is used as a method to estimate the nucleolus, the effectiveness of the clustering features should be evaluated based on their estimation performances.

\section{Evaluating Nucleolus Estimation with Stratified Random Sampling}

The proposed nucleolus estimation method involves three different clustering techniques, namely K-means clustering, Hierarchical clustering with ward linkage, and GMM with full covariance matrices. For each of these clustering techniques, two types of clustering features are investigated, namely the grand coalition cooperative energy profile, and the grand coalition marginal contribution profile. To compare these methods, a tool that is independent of all the clustering techniques is needed to evaluate the estimated nucleolus $\nu^{\text {est }}$.

The stratified random sampling strategy proposed in [24] is used as the base technique. In this approach, $\pi(\mathcal{N})$ is defined as the set of all possible permutations with player set $\mathcal{N}$, and $O: \mathcal{N} \rightarrow \mathcal{N}$ is a permutation that specifies the player assigned to position $l$ as $O(l)$. For a given $O \in \pi(\mathcal{N})$, the set of predecessors of the player $i$ is denoted as $\operatorname{Pre}^{i}(O)$, where if $i=O(l)$, then $\operatorname{Pre}^{i}(O)=\{O(1), O(2), \ldots, O(l-1)\} . \pi(\mathcal{N})$ is then divided into subpopulations, each of which is called a stratum that has the same number of predecessors for each player. A stratum, or a stratified set of player permutations, is denoted as $P_{i l}:=\{O \in \pi(\mathcal{N}) \mid O(l)=i, \forall i, l \in[1, N]\}$. Therefore, $P_{i l}$ contains every permutation $O \in \pi(\mathcal{N})$, in which player $i$ is in position $l$.

Selecting the same number of samples from each stratum ensures that all players and all sizes of coalitions are equally represented. To adapt this approach to evaluate the nucleolus estimation results, the following procedure is implemented:

1) An initial stratum sample size $m=1$ is set.

2) For each stratum $P_{i l}$, a random permutation sample $M_{i l}$ of size $m$ is obtained.

3) A set of coalitions $\Re_{m}:=\left\{\mathcal{T} \mid \mathcal{T}=\operatorname{Pre}^{i}(O) \cup i, \forall O \in\right.$ $\left.M_{i l}, \forall i, l \in[1, N]\right\}$ is defined. 
4) $v(\mathcal{T})$ is calculated using (6), $\forall \mathcal{T} \in \mathfrak{R}_{m}$. Then the coalition samples' excesses $\varepsilon_{m}\left(\boldsymbol{\nu}^{e s t}, \mathcal{T}\right), \forall \mathcal{T} \in \mathfrak{R}_{m}$ are calculated using (7). The number of positive excesses $\left|\varepsilon_{m}^{\text {pos }}\right|$ is obtained.

5) $m=m+1$, and then Step $2-4$ is repeated until $\mid \frac{\left|\varepsilon_{m}^{p o s}\right|}{\left|\mathfrak{R}_{m}\right|}-$ $\frac{\left|\varepsilon_{m-1}^{\text {pos }}\right|}{\left|\mathfrak{R}_{m-1}\right|} \mid \leq \delta$ where $\delta$ is the convergence threshold. In this study it is set to be $0.5 \%$.

\section{CASE Studies}

The model inputs in this study are as follows: the domestic load data were measured in the Customer-Led Network Revolution trials [35]; the model time frame is 24 hours starting from the midnight of a sunny summer day in July; the PV systems are $4 \mathrm{~kW}$ with fixed 20 degree tilt, simulated in PVWatts [36] using the London Gatwick solar data; the energy import price follows a UK Economy 7 residential rate structure: $£ 0.08 / \mathrm{kWh}$ for midnight-7am, and $£ 0.18 / \mathrm{kWh}$ for 7am-midnight [37], and the energy export price is the UK feed-in tariff [38] fixed at $£ 0.0379 / \mathrm{kWh}$; the ES model has an energy capacity of $7 \mathrm{kWh}$, a maximum charge power of $3.5 \mathrm{~kW}$, a maximum discharge power of $3.2 \mathrm{~kW}$, both charge and discharge efficiencies of $95 \%$, an initial state of charge of $50 \%$, and a state of charge range of $20-95 \%$.

Both the PV and ES adoption rates are fixed at 50\%, and ownerships are randomly assigned independently of each other. In other words, each prosumer can have a PV system, or an ES system, or both, or neither. Even though the majority of current ES owners also own PV systems, the model is set up this way to provide an insight into how the profit distributes among different prosumer types. Additionally, whether the ES assignment correlates with the PV assignment does not affect the grand coalition cooperative operation.

Three case studies are conducted in this section. The first case study compares the computation times under varying numbers of clusters $K$ and with different clustering techniques. The second case study demonstrates the higher accuracy of the proposed method over an existing one, and compares the estimation accuracies of different techniques with different clustering features. The third case study focuses on the trade-off between the computation time and estimation accuracy and the impact of uncertainty in large games.

\section{A. Nucleolus Estimation Computation Time}

The first case study focuses on the nucleolus computation time $^{2}$. Taking K-means clustering as an example, using the grand coalition cooperative energy profile as the clustering features, the nucleolus computation time is plotted with an increasing number of prosumers in Fig. 6. Compared to the full nucleolus computation, clustering is able to help scale up the cooperative energy management scheme to hundreds of players while limiting the computation time within 24 hours, which is the time scale of this proposed scheme.

\footnotetext{
${ }^{2}$ The models in this paper are run on a computer equipped with $16 \mathrm{~GB}$ RAM and a $2.8 \mathrm{GHz}$ Quad-Core Intel Core i5 processor.
}

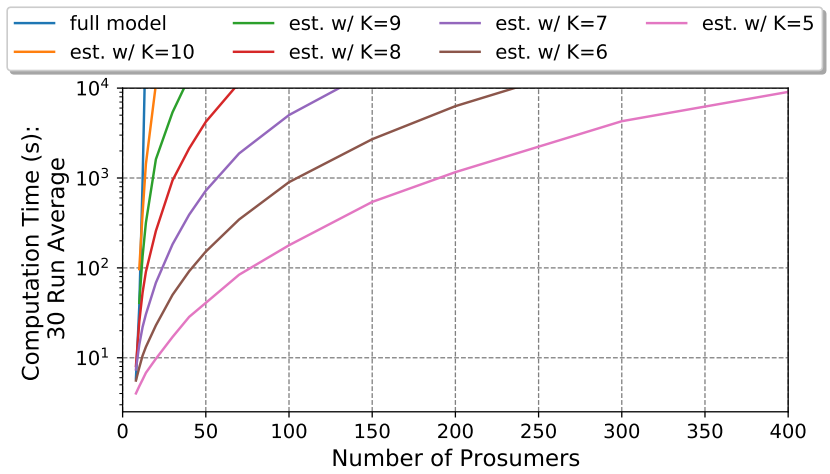

Fig. 6: Comparison of the nucleolus estimation time using K-means clustering with varying numbers of clusters

TABLE I: Clustering Computation Time (s) Avg. over 10 Runs

\begin{tabular}{cccccc}
\hline No. prosumers $(K)$ & $\mathbf{1 4}(10)$ & $\mathbf{2 0}(9)$ & $\mathbf{3 0}(8)$ & $\mathbf{5 0}(7)$ & $\mathbf{1 0 0}(6)$ \\
\hline K-means & 5.3 & 4.0 & 3.5 & 4.1 & 5.1 \\
Hierarchical-ward & 0.6 & 0.5 & 0.5 & 0.6 & 0.5 \\
GMM-full & 2.2 & 1.8 & 1.7 & 2.1 & 1.7 \\
\hline
\end{tabular}

It can be expected that the higher the $K$ value is, the more accurate the estimation would be (this is empirically validated in Section $\mathrm{V}-\mathrm{C}$ ). In order to demonstrate the contrast of results between different clustering techniques, an aggressive computation time target is set: $\leq 10^{3} \mathrm{~s}$. If up to 100 players is considered, based on Fig. 6 , the maximum $K$ that guarantees the computation time being less than $10^{3} \mathrm{~s}$ is selected. Therefore, for $14,20,30,50,100$ players, $K=10,9,8,7,6$ respectively. Table I shows the computation time for the clustering step only. Note that the computation time for K-means clustering and GMM is influenced by the number of runs repeated with different initial conditions. This number is set to be 1000 for K-means and 100 for GMM in this paper. It can be seen that although different clustering techniques require slightly different computation times, the clustering computation time is negligible compared to the rest of the nucleolus estimation process. Therefore, Fig. 6 is representative of all of the clustering techniques.

\section{B. Nucleolus Estimation Computation Accuracy}

As discussed in Section IV-A, the advantage of the proposed method is its ability to preserve the individuality of the prosumers and to estimate the nucleolus for all participating prosumers. Even though clustering is also used in the nucleolus estimation method in [21], it fails to provide a robust division of payoffs within the clustered players. For a 14-prosumer example, Fig. 7 compares the nucleolus estimation performances of the two methods, where each marker represents a participating prosumer's payoff allocations based on the full nucleolus (y-axis) and the nucleolus estimations (x-axis). Regardless of the prosumer type, the proposed nucleolus estimation method is shown to significantly outperform the clustered players method in terms of the estimation accuracy. It is worth noting that all prosumer types benefit from the scheme, and even though the prosumers with only ES systems in this example have higher payoff allocations than the other prosumer types, 


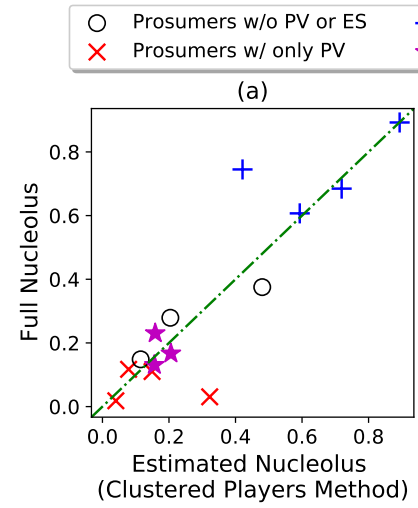

+ Prosumers w/ only ES

Prosumers w/ both PV and ES

Fig. 7: Comparison of the Nucleolus estimation performances for a 14-prosumer cooperative energy management scheme with $K=5$ : (a) full nucleolus vs. estimated nucleolus with the clustered players method in [21]; (b) full nucleolus vs. estimated nucleolus with the proposed method using K-means clustering based on the grand coalition cooperative energy profiles as the clustering features.

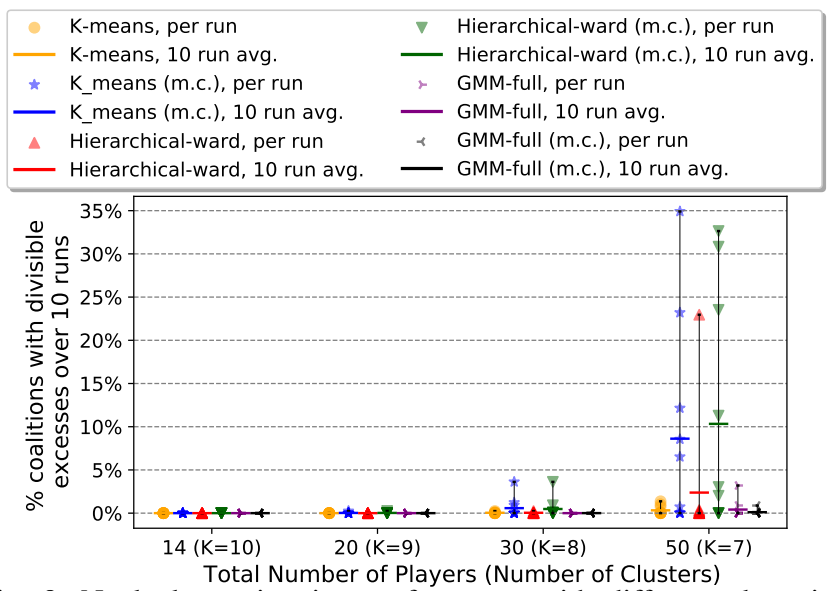

Fig. 8: Nucleolus estimation performance with different clustering techniques validated using the stratified random sampling based approach $(m=1)$.

this is not always the case. The payoff distribution among different prosumer types is influenced by factors such as the DER penetration, the prosumer overall consumption level, the energy prices, etc. How these factors affect the final payoffs is an interesting area for future work.

Under the proposed method, the estimation performances of different clustering techniques are compared based on the percentage of coalitions with divisible excesses. A divisible excess is a positive excess that, after divided by the number of prosumers within the corresponding coalition, has a rounded value greater or equal to $£ 0.01$, the smallest monetary reward that can be realized in practice. A coalition with a divisible excess can become independent and achieve enough additional profits to be allocated to all of its member prosumers.

With prosumer profiles randomly selected from [35] in each of the 10 runs, Fig. 8 compares the estimation accuracies of different clustering techniques with different clustering features. Setting $10^{3} \mathrm{~s}$ to be the upper bound of the computation time, The maximum numbers of clusters are $10,9,8,7$ for $N=14,20,30,50$ respectively, regardless of the clustering
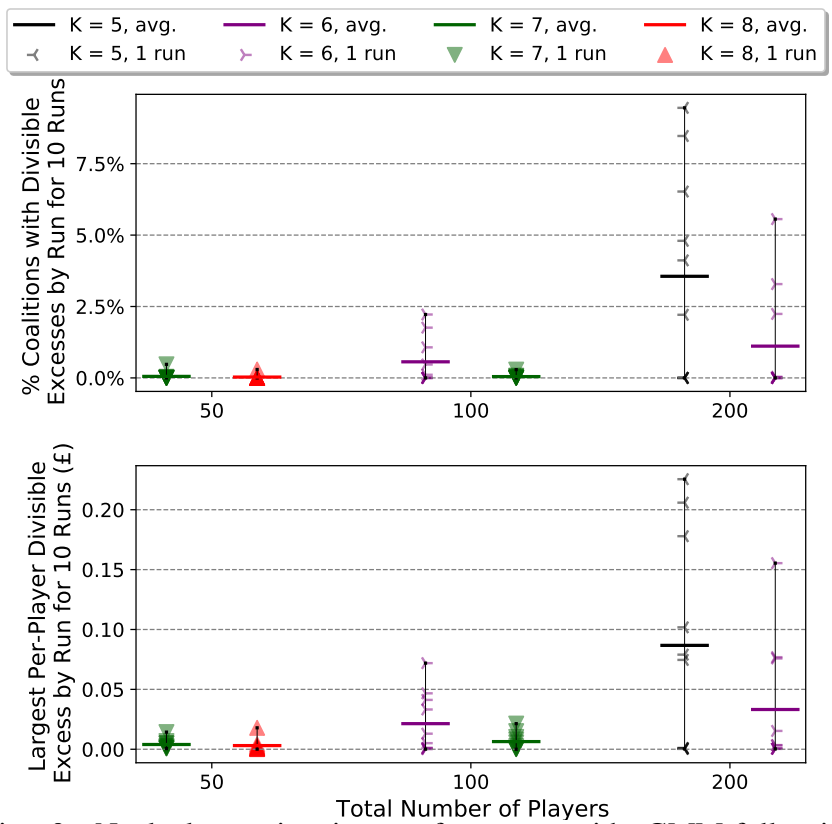

Fig. 9: Nucleolus estimation performance with GMM-full with marginal contribution feature using different $K$ values, validated using the stratified random sampling based approach $(m=1)$.

technique. It shows that for $N \leq 30$, all of the clustering techniques lead to an average of less than $1 \%$ and a maximum of less than $5 \%$ of the coalitions having divisible excesses. For $N=50$, however, hierarchical clustering with both clustering features and K-means with the marginal contribution profiles all yield an average of more than $1 \%$ and a maximum of more than $20 \%$ of coalitions with divisible excesses, while GMM with the grand coalition marginal contribution profile as the clustering features produces the best estimation results with only an average of $0.1 \%$ and a maximum of $0.9 \%$ of coalitions having divisible excesses.

\section{Scaling up with Relaxed Computation Time}

If the computation time threshold is relaxed, the estimation accuracy can be improved by increasing the number of $K$. For example, if the time limit is $10^{4} \mathrm{~s}, \mathrm{~K}$ can be increased to 7 and 6 for a 100-player and a 200-player game respectively.

Identified as the best nucleolus estimation method in Section V-B, GMM with the grand coalition marginal contribution profile as the clustering feature is applied to compare the model performances with varying $K$ values. The results are shown in Fig. 9, in which two metrics are used: 1) percentage of coalitions that have divisible excesses, which indicates the fraction of coalitions that are dissatisfied in the grand coalition; 2) the largest per-player divisible excess, which indicates the level of incentive for defection for the most dissatisfied coalition. A coalition's per-player divisible excess is obtained by dividing its divisible excesses by the number of players within the coalition. It represents the average amount of additional payoff a coalition can offer to its member prosumers after becoming independent. Although the investigation of what level of incentive can lead to defection in practice is 


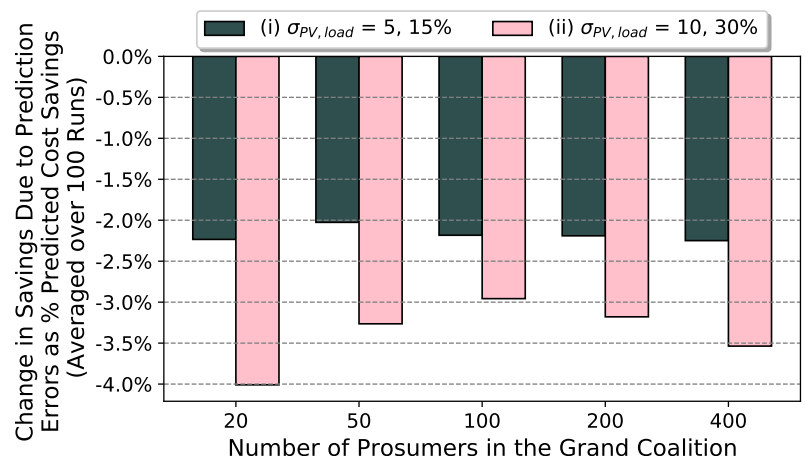

Fig. 10: The average change in profit as percentages of the total profit due the prediction errors. Over a range of numbers of prosumers, the results of two sets of standard deviations from the predicted values are shown: (i) low error case: $\sigma_{P V}=5 \%, \sigma_{\text {load }}=15 \%$; (ii) high error case: $\sigma_{P V}=10 \%, \sigma_{\text {load }}=30 \%$.

out of the scope of this paper, the closer these two values are to zero, the better the nucleolus estimation is.

Important observations can be made of Fig. 9. First, there is a trade-off between the computation time and the estimation accuracy. For a 100-player case, switching from $K=6$ to $K=7$ increases the computation time from $\sim 10^{3} \mathrm{~s}$ to $\sim 5 \times 10^{3} \mathrm{~s}$. Meanwhile, the fraction of coalitions having divisible excesses is reduced from $0.9 \%$ to $0.1 \%$, and the maximum per-player excess is reduced from $£ 0.07$ to $£ 0.02$. Second, when the same number of clusters $K$ is applied to different numbers of players $N$, increasing $N$ leads to a higher percentage of coalitions with divisible excesses and higher per-player divisible excesses, and thus worse nucleolus estimation. Third, with insufficient clusters the composition of the prosumers can have a big impact on the estimation performance. Among the 10 runs in the $N=200, K=5$ case, 4 runs yield no coalitions with divisible excesses, while one run yields $19 \%$ of coalitions with divisible excesses. An interesting extension is to investigate the types of prosumer compositions that lead to poor estimation results and the strategies to resolve this issue without increasing $K$.

It is expected that the consumption and generation prediction uncertainties may influence the model outcome. A Monte Carlo analysis is conducted on the impact of uncertainties on the total profit of the scheme. The actual consumption and generation profiles are generated assuming the value at each timestep follows a normal distribution, with the mean being the predicted value. For each model run, only one actual PV profile is generated that is followed by all PV units since they are assumed to be located close to one another, but the actual consumption profiles are individually generated for all prosumers. Using two sets of standard deviations: (i) $\sigma_{P V}=$ $5 \%, \sigma_{\text {load }}=15 \%$; (ii) $\sigma_{P V}=10 \%, \sigma_{\text {load }}=30 \%$, the model is run 100 times for each game size $(N=20,50,100,200,400)$. Fig. 10 shows the resulting changes in the grand coalition profits as percentages of the total profits. It can be seen that uncertainties can lead to a reduction in the total profit, but the reductions are only around $2 \%$ and $3-4 \%$ respectively for the low and high uncertainty cases. This reduction is insignificant mainly because the profit is measured by the cost difference between the baseline non-cooperative operation and the proposed cooperative operation, and the deviations from the expected values apply to both of these quantities.

\section{CONCLUSION}

To improve the scalability of the cooperative game theory based energy management scheme, this paper proposes a clustering based method to estimate the nucleolus, a stabilizing payoff allocation that guarantees that no player or group of players can be financially better off by defecting from the grand coalition. With $K$ clusters, the computational complexity for an $N$-player 'game' is reduced from $\sim \mathcal{O}\left(2^{N}\right)$ to $\sim \mathcal{O}\left(2^{K} N\right)$. While existing studies are only able to compute a stabilizing payoff allocation for a maximum of 15 players in such a cooperative energy management scheme, the proposed method is shown to scale it up to over 100 players, making this scheme applicable to a normal distribution feeder.

Three clustering techniques and two clustering features are applied in the proposed estimation method, and the Gaussian mixture model coupled with the marginal contribution profiles is shown to have the best estimation performance. For a 100player 'game', by grouping the prosumers into 7 clusters, the computation of the cooperative scheme including the payoff allocations to the prosumers takes less than 3 hours, which is shorter than the time needed to compute the full nucleolus of a 14-player 'game'. The nucleolus estimation leaves an average of only $0.1 \%$ of the coalitions that will have divisive excesses, the highest of which is only $£ 0.02$ per person, amounting to a very low incentive for any coalition to defect from this cooperative energy management scheme.

The proposed method has made the theoretic scheme of cooperative energy management computationally viable at a realistic scale. Additional technological challenges such as real-time communication and control of appliances, and regulatory challenges around community sharing of energy still need to be addressed for the implementation of the cooperative energy management scheme.

\section{REFERENCES}

[1] S. Bull, "Renewable energy today and tomorrow," Proceedings of the IEEE, vol. 89, no. 8, pp. 1216-1226, 2001.

[2] Y. Parag and B. K. Sovacool, "Electricity market design for the prosumer era," Nature Energy, vol. 1, no. 4, p. 16032, 2016.

[3] J. Skea, D. Anderson, T. Green, R. Gross, P. Heptonstall, and M. Leach, "Intermittent renewable generation and maintaining power system reliability," IET Generation, Transmission Distribution, vol. 2, no. 1, pp. 82-89, January 2008.

[4] K. C. Divya and J. Østergaard, "Battery energy storage technology for power systems-An overview," Electric Power Systems Research, vol. 79, no. 4, pp. 511-520, 2009.

[5] P. M. Van De Ven, N. Hegde, L. Massoulié, and T. Salonidis, "Optimal control of end-user energy storage," IEEE Transactions on Smart Grid, vol. 4, no. 2, pp. 789-797, 2013.

[6] T. Sousa, T. Soares, P. Pinson, F. Moret, T. Baroche, and E. Sorin, "Peer-to-peer and community-based markets: A comprehensive review," Renewable and Sustainable Energy Reviews, vol. 104, pp. 367 - 378, 2019.

[7] A. Pyrgou, A. Kylili, and P. A. Fokaides, "The future of the feed-in tariff (fit) scheme in europe: The case of photovoltaics," Energy Policy, vol. 95, pp. $94-102,2016$.

[8] L. Jia and L. Tong, "Dynamic pricing and distributed energy management for demand response," IEEE Transactions on Smart Grid, vol. 7, no. 2, pp. 1128-1136, March 2016. 
[9] P. Berenbrink, T. Friedetzky, L. A. Goldberg, P. W. Goldberg, Z. Hu, and R. Martin, "Distributed selfish load balancing," SIAM Journal on Computing, vol. 37, no. 4, pp. 1163-1181, 2007.

[10] T. Morstyn, A. Teytelboym, and M. D. Mcculloch, "Bilateral contract networks for peer-to-peer energy trading," IEEE Transactions on Smart Grid, vol. 10, no. 2, pp. 2026-2035, March 2019.

[11] L. Han, T. Morstyn, and M. McCulloch, "Incentivizing prosumer coalitions with energy management using cooperative game theory," IEEE Transactions on Power Systems, vol. 34, no. 1, pp. 303-313, Jan 2019.

[12] W. Saad, Z. Han, H. V. Poor, and T. Basar, "Game-theoretic methods for the smart grid: An overview of microgrid systems, demand-side management, and smart grid communications," IEEE Signal Processing Magazine, vol. 29, no. 5, pp. 86-105, Sept 2012.

[13] W. Lee, L. Xiang, R. Schober, and V. W. S. Wong, "Direct electricity trading in smart grid: A coalitional game analysis," IEEE Journal on Selected Areas in Communications, vol. 32, no. 7, pp. 1398-1411, 2014.

[14] L. Han, T. Morstyn, and M. McCulloch, "Constructing prosumer coalitions for energy cost savings using cooperative game theory," in 2018 Power Systems Computation Conference (PSCC), June 2018, pp. 1-7.

[15] A. C. Chapman, S. Mhanna, and G. Verbič, "Cooperative game theory for non-linear pricing of load-side distribution network support," The 3rd IJCAI Algorithmic Game Theory Workshop, 2017.

[16] J. K. Sankaran, "On finding the nucleolus of an n-person cooperative game," International Journal of Game Theory, vol. 19, no. 4, pp. 329338, Dec 1991.

[17] P. Chakraborty, E. Baeyens, K. Poolla, P. P. Khargonekar, and P. Varaiya, "Sharing storage in a smart grid: A coalitional game approach," IEEE Transactions on Smart Grid, vol. 10, no. 4, pp. 4379-4390, 2019.

[18] C. Feng, F. Wen, S. Member, and S. You, "Coalitional game based transactive energy management in local energy communities," IEEE Transactions on Power Systems, vol. PP, no. c, p. 1, 2019.

[19] T. Chen, K. Qian, A. Mutanen, B. Schuller, P. Jarventausta, and W. Su, "Classification of electricity customer groups towards individualized price scheme design," 2017 North American Power Symposium, NAPS 2017, no. 1, pp. 4-7, 2017.

[20] Y. Wang, Q. Chen, C. Kang, Q. Xia, and M. Luo, "Sparse and redundant representation-based smart meter data compression and pattern extraction," IEEE Transactions on Power Systems, vol. 32, no. 3, pp. 2142-2151, 2017.

[21] L. Han, T. Morstyn, C. Crozier, and M. McCulloch, "Improving the scalability of a prosumer cooperative game with k-means clustering," in 2019 IEEE Milan PowerTech, June 2019, pp. 1-6.

[22] G. Chicco, O. M. Ionel, and R. Porumb, "Formation of load pattern clusters exploiting ant colony clustering principles," IEEE EuroCon 2013, no. July, pp. 1460-1467, 2013.

[23] A. Rajabi, M. Eskandari, M. J. Ghadi, L. Li, J. Zhang, and P. Siano, "A comparative study of clustering techniques for electrical load pattern segmentation," Renewable and Sustainable Energy Reviews, no. August, 2019.

[24] J. Castro, D. Gómez, E. Molina, and J. Tejada, "Improving polynomial estimation of the Shapley value by stratified random sampling with optimum allocation," Computers and Operations Research, vol. 82, pp. 180-188, 2017.

[25] L. S. Shapley, "Cores of convex games," International Journal of Game Theory, vol. 1, no. 1, pp. 11-26, 1971.

[26] E. Baeyens, E. Y. Bitar, P. P. Khargonekar, and K. Poolla, "Coalitional aggregation of wind power," IEEE Transactions on Power Systems, vol. 28 , no. 4, pp. 3774-3784, 2013.

[27] K. Worthmann, C. M. Kellett, P. Braun, L. Grüne, and S. R. Weller, "Distributed and decentralized control of residential energy systems incorporating battery storage," IEEE Transactions on Smart Grid, vol. 6, no. 4, pp. 1914-1923, 2015.

[28] T. Morstyn, B. Hredzak, and V. G. Agelidis, "Control strategies for microgrids with distributed energy storage systems: An overview," IEEE Transactions on Smart Grid, vol. PP, no. 99, pp. 1-1, 2016.

[29] R. Li, F. Li, and N. D. Smith, "Multi-resolution load profile clustering for smart metering data," IEEE Transactions on Power Systems, vol. 31, no. 6, pp. 4473-4482, 2016.

[30] R. Li, C. Gu, F. Li, G. Shaddick, and M. Dale, "Development of low voltage network templates - part i: Substation clustering and classification," IEEE Transactions on Power Systems, vol. 30, no. 6 , pp. 3036-3044, 2015.

[31] G. Chicco, R. Napoli, and F. Piglione, "Comparisons among clustering techniques for electricity customer classification," IEEE Transactions on Power Systems, vol. 21, no. 2, pp. 933-940, May 2006.
[32] M. A. Figueiredo and A. K. Jain, "Unsupervised learning of finite mixture models," IEEE Transactions on Pattern Analysis and Machine Intelligence, vol. 24, no. 3, pp. 381-396, 2002.

[33] S. Haben, C. Singleton, and P. Grindrod, "Analysis and clustering of residential customers energy behavioral demand using smart meter data," IEEE Transactions on Smart Grid, vol. 7, no. 1, pp. 136-144, 2016.

[34] G. Chicco, "Overview and performance assessment of the clustering methods for electrical load pattern grouping," Energy, vol. 42, no. 1, pp. 68-80, 2012.

[35] Network-Revolution. (2014) Enhanced profiling of domestic customers with solar photovoltaics (pv). [Online] Available: http://www.networkrevolution.co.uk/project-library/datasettc5-enhanced-profiling-solar-photovoltaic-pv-users/

[36] Pvwatts.nrel.gov. (2019) Pvwatts calculator. [Online]. Available: http://pvwatts.nrel.gov/pvwatts.php

[37] GOV.UK. (2019, June) Domestic energy bills. [Online]. Available: https://www.gov.uk/government/collections/domestic-energy-prices

[38] GOV.UK. (2019, Feb.) Feed-in tariffs: get money for generating your own electricity. [Online]. Available: https://www.ofgem.gov.uk/environmental-programmes/fit/fit-tariff-rates

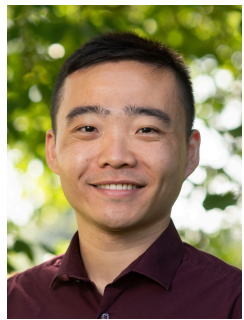

Liyang Han (S'17) received the B.S degree in energy engineering from Zhejiang University, China, in 2012, and the M.S. degree in civil engineering from Stanford University, CA, USA, in 2014. He recently finished his PhD defense at the University of Oxford and is expecting to confirm his $\mathrm{PhD}$ degree by October 2020 .

$\mathrm{He}$ joined the Energy Analytics and Markets (ELMA) group at the Technical University of Denmark (DTU) in July 2020 as a postdoctoral researcher. His research interests include prosumercentric energy markets and data markets.

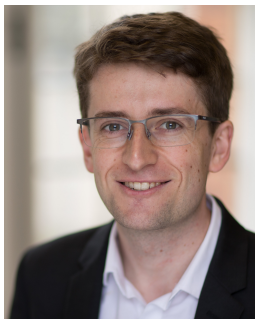

Thomas Morstyn (S'14-M'16) received the BEng (Hon.) degree from the University of Melbourne, Australia, in 2011, and the PhD degree from the University of New South Wales, Australia, in 2016, both in electrical engineering.

$\mathrm{He}$ is a Lecturer in Power Electronics and Smart Grids with the School of Engineering at the University of Edinburgh, and he is also a visiting fellow with the Oxford Martin School at the University of Oxford. His research interests include multi-agent control and market design for integrating distributed energy resources into power system operations.

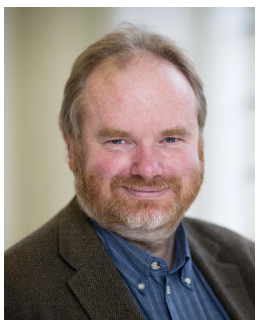

Malcolm D. McCulloch (SM'89) received the B.Sc. (Eng.) and Ph.D. degrees in electrical engineering from the University of the Witwatersrand, Johannesburg, South Africa, in 1986 and 1990, respectively.

In 1993, he joined the University of Oxford, Oxford, U.K., to head up the Energy and Power Group, where he is currently an Associate Professor with the Department of Engineering Science. He is active in the areas of electrical machines, transport, and smart grids. His work addresses transforming existing power networks, designing new power networks for the developing world, developing new technology for electric vehicles, and developing approaches to integrated mobility. 